\title{
Total Tumor RNA-loaded Dendritic Cell Vaccine
}

National Cancer Institute

\section{Source}

National Cancer Institute. Total Tumor RNA-loaded Dendritic Cell Vaccine. NCI

Thesaurus. Code C116913.

A cancer vaccine containing autologous dendritic cells (DCs) that are loaded with total tumor RNA (TTRNA) from a specific tumor, with potential immunostimulatory and antineoplastic activities. Upon administration, TTRNA-loaded DC vaccine may elicit a highly specific cytotoxic T-cell (CTL) response against the tumor-associated antigens (TAAs) encoded by the TTRNA. 